\title{
Improvements on Robust Stability of Sampled-Data System with Long Time Delay
}

\author{
Shigang Wang, ${ }^{1}$ Yongli Bi, ${ }^{1}$ and Yingsong $\mathrm{Li}^{2}$ \\ ${ }^{1}$ School of Mechanical \& Electronic Engineering, Heilongjang University, Harbin 150080, China \\ ${ }^{2}$ College of Information and Communications Engineering, Harbin Engineering University, Harbin 150001, China \\ Correspondence should be addressed to Shigang Wang; wsg6363@126.com
}

Received 24 September 2013; Accepted 5 March 2014; Published 7 April 2014

Academic Editor: Vu Ngoc Phat

Copyright (c) 2014 Shigang Wang et al. This is an open access article distributed under the Creative Commons Attribution License, which permits unrestricted use, distribution, and reproduction in any medium, provided the original work is properly cited.

\begin{abstract}
This paper mainly studies the problem of the robust stability analysis for sampled-data system with long time delay. By constructing an improved Lyapunov-Krasovskii functional and employing some free weighting matrices, some new robust stability criteria can be established in terms of linear matrix inequalities. Furthermore, the proposed equivalent criterion eliminates the effect of free weighing matrices such that numbers of decision variables and computational burden are less than some existing results. A numerical example is also presented and compared with previously proposed algorithm to illustrate the feasibility and effectiveness of the developed results.
\end{abstract}

\section{Introduction}

In the last few decades, there has been much interest in long time delay system. This is due to its key role in theory research and practical application, such as welding industry, communication networks, and electrical power system. Lots of relevant research results to long time delay systems have been reported in the literature. To mention a few, modeling of long time delay system was considered in [1]; analysis and synthesis results of such system were reported in [2-8]; the fault detection and filtering problem were solved in $[9,10]$. However, relationship between sampling period and time delay in the plant is not fully considered [11, 12], which has inevitably limited the applicability of the aforementioned resulted.

In the recent years, increasing attention has been devoted to the problem of sampled-data system with long time delay, in which time delay of the plant is usually longer than a sampling period. When such problem is researched in the discrete-time framework, concentrated augmentation approach and direct distribution approach can be chosen. However, in concentrated augmentation approach, time delay is not taken into consideration in process of deriving stability criterion and designing desired controller $[5,6,13]$. Therefore, this approach is usually regarded as being more numbers of decision variable and computational burden than direct distribution approach.

In this paper, we make an attempt to solve the robust stability problem of sampled-data systems with long time delay. Some free weighting matrices and an equivalent criterion are introduced, in order to reduce numbers of decide variable and computation burden. Numerical examples are also presented to illustrate the feasibility and effectiveness of the developed results.

\section{Problem Formulation}

Consider a continuous plant of sampled-data system with long time delay:

$$
\begin{aligned}
\mathcal{S}: \dot{x}(t) & =A_{0} x(t)+A_{1} x(t-\tau), \\
x(t) & =x_{0}, \quad t \in[-\tau, 0],
\end{aligned}
$$

where $x(t) \in \mathbb{R}^{n}$ is the state vector and $A_{0}$ and $A_{1}$ are the constant matrices of appropriate dimensions. $x_{0}$ is the initial state vector.

Assumption 1. Sampler is time-driven with a constant sampling period $h(h>0)$. 
Assumption 2. The time delay $\tau$ is uncertain but is evaluated between two adjacent sampling periods; namely, $(m-1) h \leq$ $\tau \leq m h$, where $m>1$ is a known constant.

Lemma 3 (see [14]). For given matrices $Q=Q^{\mathrm{T}}, \mathscr{H}$, and $\mathscr{E}$, with appropriate dimensions,

$$
Q+\mathscr{H} \mathscr{F}(k) \mathscr{E}+\mathscr{E}^{\mathrm{T}} \mathscr{F}^{\mathrm{T}}(k) \mathscr{H}^{\mathrm{T}}<0
$$

holds for all $\mathscr{F}(k)$ satisfying $\mathscr{F}^{\mathrm{T}}(k) \mathscr{F}(k) \leq I$ if and only if there exists $\varepsilon>0$ :

$$
\mathcal{Q}+\varepsilon \mathscr{H}^{\mathrm{T}}+\varepsilon^{-1} \mathscr{E}^{\mathrm{T}} \mathscr{E}<0 .
$$

\section{Main Results}

Discretizing system (1) in one period, we can obtain the discrete state equation of sampled-data system:

$$
\begin{gathered}
\mathcal{U}: x(k+1)=G_{0} x(k)+G_{1} x(k-m+1)+G_{2} x(k-m), \\
x(k)=x_{0}, \quad k=-m,-m+1, \ldots, 0,
\end{gathered}
$$

where

$$
\begin{aligned}
& G_{0}=e^{A_{0} h}, \\
& G_{1}=\int_{0}^{m h-\tau} e^{A_{0} t} d t A_{1}, \\
& G_{2}=\int_{m h-\tau}^{h} e^{A_{0} t} d t A_{1} .
\end{aligned}
$$

Let

$$
\begin{aligned}
\bar{G}_{1} & =-L \operatorname{diag}\left\{\frac{1}{\lambda_{1}}, \ldots, \frac{1}{\lambda_{n}}\right\} L^{-1} A_{1}, \\
\bar{G}_{2} & =L \operatorname{diag}\left\{\frac{1}{\lambda_{1}} e^{\lambda_{1} h}, \ldots, \frac{1}{\lambda_{n}} e^{\lambda_{n} h}\right\} L^{-1} A_{1}, \\
D & =L \operatorname{diag}\left\{\frac{1}{\lambda_{1}} e^{\lambda_{1} \beta_{1}}, \ldots, \frac{1}{\lambda_{n}} e^{\lambda_{n} \beta_{1}}\right\}, \\
F(\tau) & =\operatorname{diag}\left\{e^{\lambda_{1}\left(m h-\tau-\beta_{1}\right)}, \ldots, e^{\lambda_{n}\left(m h-\tau-\beta_{n}\right)}\right\}, \\
E & =L^{-1} A_{1} .
\end{aligned}
$$

Then

$$
G_{1}(\tau)=\bar{G}_{1}+D F(\tau) E .
$$

In the same way, we can get

$$
G_{2}(\tau)=\bar{G}_{2}-D F(\tau) E .
$$

Choosing appropriate variables $\beta_{1}, \beta_{2}, \ldots, \beta_{n}$, so that they satisfy $e^{\lambda_{i}\left(m h-\tau-\beta_{i}\right)} \leq 1$, then

$$
F^{\mathrm{T}}(\tau) F(\tau) \leq I
$$

Remark 4. Modeling of sampled-data system with long time delay has been reported in [13].
3.1. Stability Condition. The stability condition presented in this section is based on system (4); we will proceed with the following theorem.

Theorem 5. System (4) is asymptotically stable if there exist matrices $P_{1}>0, S_{1}>0, S_{2}>0, Q_{1}>0, Q_{2}>0, R_{1}>0$, $R_{2}>0, M, L$, and $N$, satisfying

$$
\left[\begin{array}{cc}
\Pi_{1}+\Pi_{2}+\Pi_{2}^{\mathrm{T}}+\Pi_{3} & \Pi_{4} \\
* & \Pi_{5}
\end{array}\right]<0
$$

where

$$
\begin{aligned}
& \Pi_{1} \\
& =\left[\begin{array}{ccc}
G_{0}^{\mathrm{T}} P_{1} G_{0}-P_{1}+S_{1}+S_{2} & G_{0}^{\mathrm{T}} P_{1} G_{1} & G_{0}^{\mathrm{T}} P_{1} G_{2} \\
+(m-1) Q_{1}+m Q_{2} & G_{1}^{\mathrm{T}} P_{1} G_{1}-S_{1} & G_{1}^{\mathrm{T}} P_{1} G_{2} \\
* & * & G_{2}^{\mathrm{T}} P_{1} G_{2}-S_{2}
\end{array}\right],
\end{aligned}
$$

$$
\begin{aligned}
& \Pi_{2}=\left[\begin{array}{lll}
M+N & L-M & -L-N
\end{array}\right], \\
& \Pi_{3}=m\left[\begin{array}{lll}
G_{0}-I & G_{1} & G_{2}
\end{array}\right]^{\mathrm{T}}\left(R_{1}+R_{2}\right)\left[\begin{array}{lll}
G_{0}-I & G_{1} & G_{2}
\end{array}\right], \\
& \Pi_{4}=\left[\begin{array}{lll}
\sqrt{m-1} M & L & \sqrt{m} N
\end{array}\right], \\
& \Pi_{5}=\operatorname{diag}\left\{\begin{array}{lll}
-R_{1} & -R_{1} & \left.-R_{2}\right\} .
\end{array}\right.
\end{aligned}
$$

Proof. Letting

$$
\eta(k)=x(k+1)-x(k)
$$

then

$$
\begin{gathered}
x(k+1)=x(k)+\eta(k), \\
x(k)-x(k-m+1)=\sum_{i=k-m+1}^{k-1} \eta(i), \\
x(k)-x(k-m)=\sum_{i=k-m}^{k-1} \eta(i) .
\end{gathered}
$$

Choosing a Lyapunov function candidate as

$$
\begin{aligned}
& V=V_{1}+V_{2}+V_{3}+V_{4}+V_{5}+V_{6}, \\
& V_{1}=x^{\mathrm{T}}(k) P_{1} x(k), \\
& V_{2}=\sum_{i=k-m+1}^{k-1} x^{\mathrm{T}}(i) S_{1} x(i),
\end{aligned}
$$




$$
\begin{aligned}
& V_{3}=\sum_{i=k-m}^{k-1} x^{\mathrm{T}}(i) S_{2} x(i), \\
& V_{4}=\sum_{j=-m+1}^{-1} \sum_{i=k+j}^{k-1} x^{\mathrm{T}}(i) Q_{1} x(i), \\
& V_{5}=\sum_{j=-m}^{-1} \sum_{i=k+j}^{k-1} x^{\mathrm{T}}(i) Q_{2} x(i), \\
& V_{6}=\sum_{j=-m}^{-1} \sum_{i=k+j}^{k-1} \eta^{\mathrm{T}}(i)\left(R_{1}+R_{2}\right) \eta(i),
\end{aligned}
$$

where $P_{1}=P_{1}^{\mathrm{T}}>0, S_{i}=S_{i}^{\mathrm{T}} \geq 0, i=1,2$, and $R_{i}=R_{i}^{\mathrm{T}} \geq 0$, $i=1,2$, are undetermined.

Defining $\Delta V=V(k+1)-V(k)$, and then along the solution of (4), we have

$$
\begin{aligned}
& \Delta V_{1}=x^{\mathrm{T}}(k+1) P_{1} x(k+1)-x^{\mathrm{T}}(k) P_{1} x(k) \\
& \Delta V_{2} \leq x^{\mathrm{T}}(k) S_{1} x(k)-x^{\mathrm{T}}(k-m+1) S_{1} x(k-m+1) \\
& +\sum_{j=k-m+1}^{k-1} x^{\mathrm{T}}(j) Q_{1} x(j) \\
& \Delta V_{3} \leq x^{\mathrm{T}}(k) S_{2} x(k)-x^{\mathrm{T}}(k-m) S_{2} x(k-m) \\
& +\sum_{j=k-m}^{k-1} x^{\mathrm{T}}(j) Q_{2} x(j) \\
& \Delta V_{4}=(m-1) x^{\mathrm{T}}(k) Q_{1} x(k)-\sum_{j=k-m+1}^{k-1} x^{\mathrm{T}}(j) Q_{1} x(j), \\
& \Delta V_{5}=m x^{\mathrm{T}}(k) Q_{2} x(k)-\sum_{j=k-m}^{k-1} x^{\mathrm{T}}(j) Q_{2} x(j), \\
& \Delta V_{6}=m \eta^{\mathrm{T}}(k)\left(R_{1}+R_{2}\right) \eta(k)-\sum_{l=k-m}^{k-1} \eta^{\mathrm{T}}(l)\left(R_{1}+R_{2}\right) \eta(l) \\
& =m \eta^{\mathrm{T}}(k)\left(R_{1}+R_{2}\right) \eta(k)-\sum_{l=k-m+1}^{k-1} \eta^{\mathrm{T}}(l) R_{1} \eta(l) \\
& -\sum_{l=k-m}^{k-m} \eta^{\mathrm{T}}(l) R_{1} \eta(l)-\sum_{l=k-m}^{k-1} \eta^{\mathrm{T}}(l) R_{2} \eta(l) .
\end{aligned}
$$

Defining $\xi(k)=\left[\begin{array}{lll}x^{\mathrm{T}}(k) & x^{\mathrm{T}}(k-m+1) & x^{\mathrm{T}}(k-m)\end{array}\right]^{\mathrm{T}}$, then we have

$$
\begin{aligned}
\Delta V \leq & {\left[G_{0} x(k)+G_{1} x(k-m+1)+G_{2} x(k-m)\right]^{\mathrm{T}} P_{1} } \\
& \times\left[G_{0} x(k)+G_{1} x(k-m+1)+G_{2} x(k-m)\right] \\
& -x^{\mathrm{T}}(k) P_{1} x(k)+x^{\mathrm{T}}(k) S_{1} x(k)
\end{aligned}
$$

$$
\begin{aligned}
& -x^{\mathrm{T}}(k-m+1) S_{1} x(k-m+1) \\
& +x^{\mathrm{T}}(k) S_{2} x(k)-x^{\mathrm{T}}(k-m) S_{2} x(k-m) \\
& +(m-1) x^{\mathrm{T}}(k) Q_{1} x(k)+m x^{\mathrm{T}}(k) Q_{2} x(k) \\
& +m\left[\left(G_{0}-I\right) x(k)+G_{1} x(k-m+1)+G_{2} x(k-m)\right]^{\mathrm{T}} \\
& \times\left(R_{1}+R_{2}\right) \\
& \times\left[\left(G_{0}-I\right) x(k)+G_{1} x(k-m+1)+G_{2} x(k-m)\right] \\
& -\sum_{l=k-m+1}^{k-1} \eta^{\mathrm{T}}(l) R_{1} \eta(l)-\sum_{l=k-m}^{k-m} \eta^{\mathrm{T}}(l) R_{1} \eta(l) \\
& -\sum_{l=k-m}^{k-1} \eta^{\mathrm{T}}(l) R_{2} \eta(l) \\
& +2 \xi^{\mathrm{T}}(k) M\left[x(k)-x(k-m+1)-\sum_{l=k-m+1}^{k-1} \eta(l)\right] \\
& +2 \xi^{\mathrm{T}}(k) L\left[x(k-m+1)-x(k-m)-\sum_{l=k-m}^{k-m} \eta(l)\right] \\
& +2 \xi^{\mathrm{T}}(k) N\left[x(k)-x(k-m)-\sum_{l=k-m}^{k-1} \eta(l)\right] \\
& \leq \xi^{\mathrm{T}}(k)\left[\Pi_{1}+\Pi_{2}+\Pi_{2}^{\mathrm{T}}+\Pi_{3}+(m-1) M R_{1}^{-1} M^{\mathrm{T}}\right. \\
& \left.+L R_{1}^{-1} L^{\mathrm{T}}+m N R_{2}^{-1} N^{\mathrm{T}}\right] \xi(k) \\
& -\sum_{l=k-m+1}^{k-1}\left[\xi^{\mathrm{T}}(k) M+\eta^{\mathrm{T}}(l) R_{1}\right] \\
& \times R_{1}^{-1}\left[M^{\mathrm{T}} \xi(k)+R_{1} \eta(l)\right] \\
& -\sum_{l=k-m}^{k-m}\left[\xi^{\mathrm{T}}(k) L+\eta^{\mathrm{T}}(l) R_{1}\right] \\
& \times R_{1}^{-1}\left[L^{\mathrm{T}} \xi(k)+R_{1} \eta(l)\right] \\
& -\sum_{l=k-m}^{k-1}\left[\xi^{\mathrm{T}}(k) N+\eta^{\mathrm{T}}(l) R_{2}\right] \\
& \times R_{2}^{-1}\left[N^{\mathrm{T}} \xi(k)+R_{2} \eta(l)\right] .
\end{aligned}
$$

Since $R_{i}>0, i=1,2$, the last three terms are all nonpositive. By the Schur complement [15], inequality (10) guarantees $\Pi_{1}+\Pi_{2}+\Pi_{2}^{\mathrm{T}}+\Pi_{3}+(m-1) M R_{1}^{-1} M^{\mathrm{T}}+S R_{1}^{-1} S^{\mathrm{T}}+$ $m N R_{2}^{-1} N^{\mathrm{T}}<0$. From (17), we can attain $\Delta V<-\varepsilon\|x(k)\|^{2}$, for a sufficiently small $\varepsilon$, and $x(k) \neq 0$, and the asymptotic stability is established.

3.2. Robust Stability Condition. There exist uncertainties in the stability condition of Theorem 5 . In this section, we will 
cope with uncertainties of stability condition (10), such that robust stability condition is derived.

Theorem 6. System (4) is asymptotically stable if there exist matrices $P_{1}>0, S_{1}>0, S_{2}>0, Q_{1}>0, Q_{2}>0, R_{1}>0$, $R_{2}>0, M, L$, and $N$, satisfying

$$
\left[\begin{array}{cccc}
\widetilde{\Pi}_{1}+\widetilde{\Pi}_{2} & * & * & * \\
\widetilde{\Pi}_{4}^{\mathrm{T}} & \widetilde{\Pi}_{5} & * & * \\
\widetilde{G}^{2} & \mathbf{0}_{3 \times 3} & \widetilde{\Pi}_{6} & * \\
\widetilde{\Pi}_{7} & \mathbf{0}_{2 \times 3} & \widetilde{\Pi}_{8} & \widetilde{\Pi}_{9}
\end{array}\right]<0
$$

where

$$
\begin{aligned}
& \widetilde{\Pi}_{1}=\left[\begin{array}{ccc}
-X+W_{1}+W_{2} & & \\
+(m-1) W_{3}+m W_{4} & * & * \\
0 & -S_{1} & * \\
0 & 0 & -S_{2}
\end{array}\right], \\
& \widetilde{\Pi}_{2}=\operatorname{diag}\left\{P_{1}^{-1}, I, I\right\} \times\left\{\Pi_{2}+\Pi_{2}^{\mathrm{T}}\right\} \times \operatorname{diag}\left\{P_{1}^{-1}, I, I\right\} \text {, } \\
& \widetilde{\Pi}_{4}^{\mathrm{T}}=\operatorname{diag}\left\{R_{1}^{-1}, R_{1}^{-1}, R_{2}^{-1}\right\} \times \Pi_{4}^{\mathrm{T}} \times \operatorname{diag}\left\{P_{1}^{-1}, I, I\right\}, \\
& \widetilde{G}=\left[\begin{array}{ccc}
G_{0} X & \bar{G}_{1} & \bar{G}_{2} \\
\sqrt{m}\left(G_{0} X-X\right) & \sqrt{m} \bar{G}_{1} & \sqrt{m} \bar{G}_{2} \\
\sqrt{m}\left(G_{0} X-X\right) & \sqrt{m} \bar{G}_{1} & \sqrt{m} \bar{G}_{2}
\end{array}\right], \\
& \widetilde{\Pi}_{5}=\left[\begin{array}{ccc}
-\widetilde{R}_{1} & * & * \\
0 & -\widetilde{R}_{1} & * \\
0 & 0 & -\widetilde{R}_{2}
\end{array}\right] \text {, } \\
& \widetilde{\Pi}_{6}=\left[\begin{array}{cccccc}
0 & 0 & 0 & -X & * & * \\
0 & 0 & 0 & 0 & -\widetilde{R}_{1} & * \\
0 & 0 & 0 & 0 & 0 & -\widetilde{R}_{2}
\end{array}\right] \text {, } \\
& \widetilde{\Pi}_{7}=\left[\begin{array}{ccc}
0 & 0 & 0 \\
0 & E_{1} & -E_{1}
\end{array}\right] \text {, } \\
& \widetilde{\Pi}_{8}=\left[\begin{array}{cccccc}
0 & 0 & 0 & \varepsilon_{1} D^{\mathrm{T}} & \varepsilon_{1} D^{\mathrm{T}} & \varepsilon_{1} D^{\mathrm{T}} \\
0 & 0 & 0 & 0 & 0 & 0
\end{array}\right] \\
& \widetilde{\Pi}_{9}=\left[\begin{array}{cc}
-\varepsilon_{1} I & * \\
0 & -\varepsilon_{1} I
\end{array}\right] \text {, } \\
& X=P^{-1}, \quad \widetilde{R}_{1}=R_{1}^{-1}, \quad \widetilde{R}_{2}=R_{2}^{-1}, \\
& W_{1}=X S_{1} X, \quad W_{2}=X S_{2} X, \quad W_{3}=X Q_{1} X, \quad W_{4}=X Q_{2} X .
\end{aligned}
$$

Proof. With Theorem 5, the system $\mathcal{U}$ in (4) is asymptotically stable, if there exist matrices $P_{1}, S_{i}, Q_{i}$, and $R_{i}, i=1,2$, satisfying

$$
\left[\begin{array}{ccc}
\breve{\Pi}_{1}+\Pi_{2}+\Pi_{2}^{\mathrm{T}} & * & * \\
\Pi_{4}^{\mathrm{T}} & \breve{\Pi}_{5} & * \\
\widetilde{G} & \mathbf{0}_{3 \times 3} & \widetilde{\Pi}_{6}
\end{array}\right]<0
$$

where

$$
\begin{aligned}
\breve{\Pi}_{1} & =\left[\begin{array}{ccc}
-P_{1}+S_{1}+S_{2} & 0 & 0 \\
+(m-1) Q_{1}+m Q_{2} & & -S_{1} \\
0 & 0 \\
0 & 0 & -S_{2}
\end{array}\right], \\
\breve{\Pi}_{5} & =\left[\begin{array}{ccc}
-R_{1} & * & * \\
0 & -R_{1} & * \\
0 & 0 & -R_{2}
\end{array}\right], \\
\breve{G} & =\left[\begin{array}{ccc}
G_{0} & G_{1} & G_{2} \\
G_{0}-I & G_{1} & G_{2} \\
G_{0}-I & G_{1} & G_{2}
\end{array}\right] .
\end{aligned}
$$

Substitution of the matrices $G_{1}$ and $G_{2}$ defined in (7) (8) leads to

$$
\left[\begin{array}{ccc}
\breve{\Pi}_{1}+\Pi_{2}+\Pi_{2}^{\mathrm{T}} & * & * \\
\Pi_{4}^{\mathrm{T}} & \breve{\Pi}_{5} & * \\
\widetilde{G} & \mathbf{0}_{3 \times 3} & \widetilde{\Pi}_{6}
\end{array}\right]+\theta_{1} F(\tau) \theta_{2}+\theta_{2}^{\mathrm{T}} F^{\mathrm{T}}(\tau) \theta_{1}^{\mathrm{T}}<0,
$$

where

$$
\begin{aligned}
\widehat{G} & =\left[\begin{array}{cccc}
G_{0} & \bar{G}_{1} & \bar{G}_{2} \\
\sqrt{m}\left(G_{0}-I\right) & \sqrt{m} \bar{G}_{1} & \sqrt{m} \bar{G}_{2} \\
\sqrt{m}\left(G_{0}-I\right) & \sqrt{m} \bar{G}_{1} & \sqrt{m} \bar{G}_{2}
\end{array}\right], \\
\theta_{1} & =\left[\begin{array}{lllllllll}
0 & 0 & 0 & 0 & 0 & 0 & D^{\mathrm{T}} & D^{\mathrm{T}} & D^{\mathrm{T}}
\end{array}\right]^{\mathrm{T}}, \\
\theta_{2} & =\left[\begin{array}{lllllllll}
0 & E & -E & 0 & 0 & 0 & 0 & 0 & 0
\end{array}\right] .
\end{aligned}
$$

By Lemma 3, we readily obtain

$$
\left[\begin{array}{cccc}
\breve{\Pi}_{1}+\Pi_{2}+\Pi_{2}^{\mathrm{T}} & * & * & * \\
\Pi_{4}^{\mathrm{T}} & \breve{\Pi}_{5} & * & * \\
\widetilde{G} & \mathbf{0}_{3 \times 3} & \widetilde{\Pi}_{6} & * \\
\widetilde{\Pi}_{7} & \mathbf{0}_{2 \times 3} & \bar{\Pi}_{8} & \Pi_{9}
\end{array}\right]<0,
$$

where

$$
\begin{aligned}
& \breve{\Pi}_{7}=\left[\begin{array}{ccc}
0 & 0 & 0 \\
0 & E & -E
\end{array}\right],
\end{aligned}
$$

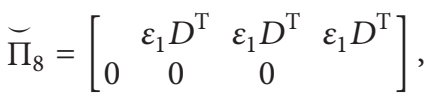

$$
\begin{aligned}
& \breve{\Pi}_{9}=\left[\begin{array}{cc}
-\varepsilon_{1} I & * \\
0 & -\varepsilon_{1} I
\end{array}\right] \text {. }
\end{aligned}
$$

A congruence transformation is applied to (24), by $\operatorname{diag}\left\{P_{1}^{-1}, I, I, R_{1}^{-1}, R_{1}^{-1}, R_{2}^{-1}, I, I, I\right\}$, we can attain LMI (18).

Fact 1. Definiteness of a matrix is invariant under congruent transformation by a full rank matrix. For instance, if $Q=$ $Q^{T} \in \mathbb{R}^{n \times n}$, and $V \in \mathbb{R}^{n \times n}$ is of full rank, then $Q>0 \rightarrow$ $V Q V^{\mathrm{T}}>0$. 
Theorem 7. System (4) is asymptotically stable if there exist real symmetric positive definite matrices $P_{1}>0, S_{i}>0, Q_{i}>0$, $R_{i}>0, i=1,2$, and matrices $L, M$, and $N$, such that the following LMI holds:

$$
\Pi_{1}+\Gamma_{1}+\Pi_{3}<0
$$

where

$$
\begin{aligned}
\Gamma_{1}= & \left(e_{1}-e_{2}\right)^{\mathrm{T}}\left(-\frac{R_{1}}{\sqrt{m-1}}\right)\left(e_{1}-e_{2}\right) \\
& +\left(e_{2}-e_{3}\right)^{\mathrm{T}}\left(-R_{1}\right)\left(e_{2}-e_{3}\right) \\
& +\left(e_{1}-e_{3}\right)^{\mathrm{T}}\left(-\frac{R_{2}}{\sqrt{m}}\right)\left(e_{1}-e_{3}\right)
\end{aligned}
$$

and $e_{i}, i=1$ to 3 , are block entry matrices of appropriate dimensions; namely, $e_{1}=\left[\begin{array}{lll}I & 0 & 0\end{array}\right], e_{2}=\left[\begin{array}{lll}0 & I & 0\end{array}\right]$, and $e_{3}=$ $\left[\begin{array}{lll}0 & 0 & I\end{array}\right]$.

Proof. Consider a nonsingular transformation matrix $\Lambda$. Congruent transformation of the LMI given in (10) with $\Lambda$ yields the following equivalent criterion:

$$
\Lambda\left[\begin{array}{cc}
\Pi_{1}+\Pi_{2}+\Pi_{2}^{\mathrm{T}}+\Pi_{3} & \Pi_{4} \\
* & \Pi_{5}
\end{array}\right] \Lambda^{\mathrm{T}}=\left[\begin{array}{cc}
\Pi_{1}+\Gamma_{1}+\Pi_{3} & \Gamma_{2} \\
* & \Gamma_{3}
\end{array}\right]<0,
$$

$$
\begin{aligned}
& \Lambda=\left[\begin{array}{cccccc}
I & 0 & 0 & -\frac{I}{\sqrt{m-1}} & 0 & -\frac{I}{\sqrt{m}} \\
0 & I & 0 & \frac{I}{\sqrt{m-1}} & -I & 0 \\
0 & 0 & I & 0 & I & \frac{I}{\sqrt{m}} \\
0 & 0 & 0 & \frac{I}{\sqrt{m-1}} & 0 & 0 \\
0 & 0 & 0 & 0 & I & 0 \\
0 & 0 & 0 & 0 & 0 & \frac{I}{\sqrt{m}}
\end{array}\right], \\
& \Gamma_{2}=\left[\begin{array}{ccc}
M_{1}+\frac{R_{1}}{m-1} & S_{1} & N_{1}+\frac{R_{2}}{m} \\
M_{2}-\frac{R_{1}}{m-1} & S_{2}+R_{1} & N_{2} \\
M_{3} & S_{3}-R_{1} & N_{3}-\frac{R_{2}}{m}
\end{array}\right], \\
& \Gamma_{3}=\operatorname{diag}\left\{-\frac{R_{1}}{m-1}-R_{1}-\frac{R_{2}}{m}\right\} .
\end{aligned}
$$

Using Schur complement, we can express the right-side LMI of (28) as follows:

$$
\Pi_{1}+\Gamma_{1}+\Pi_{3}-\Gamma_{2} \Gamma_{3}^{-1} \Gamma_{2}^{\mathrm{T}}<0
$$

Since $\Gamma_{3}<0$ holds, we can write the equivalent stability criterion as $\Pi_{1}+\Gamma_{1}+\Pi_{3}<0$, if we let the slack variable matrices $M, L$, and $N$ be as follows:

$$
M=\left[\begin{array}{c}
-\frac{R_{1}}{m-1} \\
\frac{R_{1}}{m-1} \\
0
\end{array}\right], \quad L=\left[\begin{array}{c}
0 \\
-R_{1} \\
R_{1}
\end{array}\right], \quad N=\left[\begin{array}{c}
-\frac{R_{2}}{m} \\
0 \\
\frac{R_{2}}{m}
\end{array}\right] .
$$

Hence, $\Pi_{1}+\Gamma_{1}+\Pi_{3}<0$ is equivalent to (10). In other words, Theorem 6 is equivalent to Theorem 5 .

Remark 8. Theorem 7 presents an equivalent condition for the solvability of the stability condition problem for sampleddata system with long time delay. We note that $\Pi_{1}$ and $\Pi_{3}$ include norm-bounded uncertain parameters, such that (26) cannot be solved directly. In order to solve this problem, Theorem 9 is derived as follows.

Theorem 9. System (4) is asymptotically stable if there exist real symmetric positive definite matrices $P_{1}>0, S_{i}>0, Q_{i}>0$, $R_{i}>0, i=1,2$, such that the following LMI holds:

$$
\left[\begin{array}{ccc}
\breve{\Pi}_{1}+\Gamma_{1} & * & * \\
\widehat{G} & \widetilde{\Pi}_{6} & * \\
\breve{\Pi}_{7} & \breve{\Pi}_{8} & \breve{\Pi}_{10}
\end{array}\right]<0
$$

where

$$
\breve{\Pi}_{10}=\left[\begin{array}{cc}
-\varepsilon_{2} I & * \\
0 & -\varepsilon_{2} I
\end{array}\right] .
$$

Proof. The process of proof is similar to Theorem 6, so it is omitted.

Remark 10. The equivalent stability criterion presented in Theorems 7 and 9 has no free weighting matrices, and they have only the matrix variables that are associated in the Lyapunov-Krasovskii function. Therefore, as the total number of matrix variables involved with the proposed equivalent criterion in minimum, it has less offline computational burden compared to Theorem 5. To highlight this aspect, we compare the number of decision variables involved in both the stability criterion in Table 1.

\section{Numerical Example}

Considering the system $\mathcal{S}$ in (1)

$$
A_{0}=\left[\begin{array}{cc}
-2.6 & 5.0 \\
-3.6 & -2.6
\end{array}\right], \quad A_{1}=\left[\begin{array}{cc}
-1 & 0.5 \\
1 & 0.1
\end{array}\right] \text {. }
$$




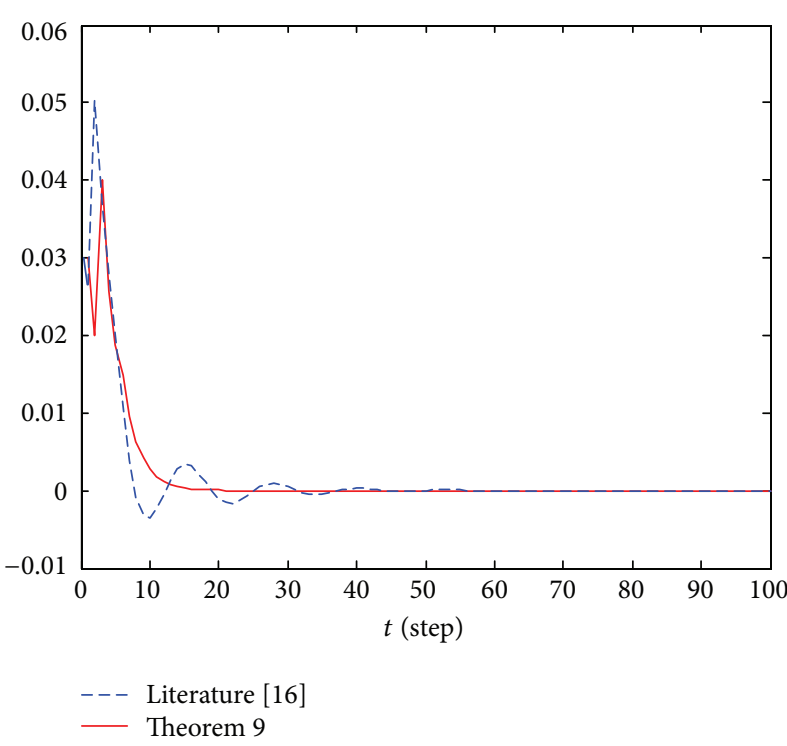

(a) $x_{1}$

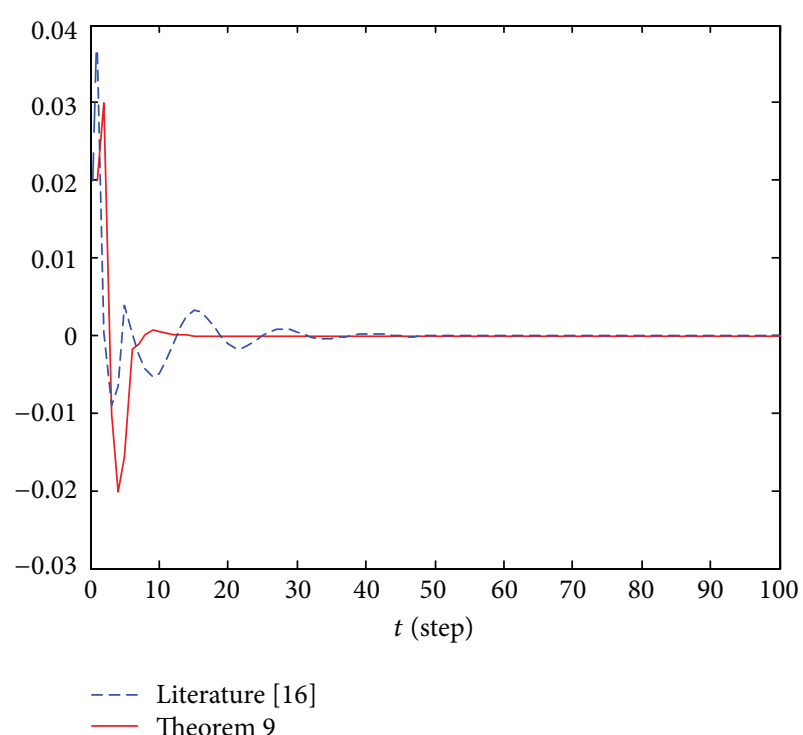

(b) $x_{2}$

FIgURE 1: State response curve of $x_{1}, x_{2}$.

TABLE 1: Comparison of decision variables.

\begin{tabular}{lc}
\hline Revelent theorem & Number of decision variables \\
\hline By [6] & $18 n^{2}+3 n$ \\
By Theorem 5 & $12.5 n^{2}+3.5 n$ \\
By Theorem 7 & $3.5 n^{2}+3.5 n$ \\
\hline
\end{tabular}

Letting $h=0.1, m=2$, and discretizing system (1), new parameter of discrete-time system is attained:

$$
\begin{array}{rlr}
G_{0} & =\left[\begin{array}{cc}
0.0345 & 0.4726 \\
-0.3403 & 0.0345
\end{array}\right], & \bar{G}_{1}=\left[\begin{array}{cc}
2.923 & -0.302 \\
-0.150 & -0.083
\end{array}\right], \\
\bar{G}_{2}=\left[\begin{array}{cc}
-2.905 & 0.317 \\
0.502 & 0.047
\end{array}\right], & \\
D & =\left[\begin{array}{cc}
0.345 & 0 \\
0 & 0.345
\end{array}\right], & E=\left[\begin{array}{ll}
-0.020 & 0.015 \\
-1.050 & 0.033
\end{array}\right] .
\end{array}
$$

Let

$$
x(0)=\left[\begin{array}{ll}
0.03 & 0.02
\end{array}\right]^{\mathrm{T}} \text {. }
$$

Remark 11. The problem of relevant norm-bounded uncertain parameter is further solved in Theorem 9. Compared with [16], the number of decision variables is $3.5 n^{2}+3.5 n$, which is less than $20.5 n^{2}+4.5 n$. Moreover, state response curve of Theorem 9's method has smaller overshoot and quicker convergence speed, which is shown in Figure 1.

\section{Conclusions}

In this correspondence paper, we have presented an equivalent stability criterion with less number of LMI variables for a robust stability criterion reported in Theorem 6 . By employing congruent transformation with nonsingular matrix, the free weighting matrices in the existing criterion are eliminated without sacrificing the conservation of stability criterion; this, in turn, yields an equivalent criterion that is mathematically less complex and computationally less expensive.

\section{Conflict of Interests}

The authors declare that there is no conflict of interests regarding the publication of this paper.

\section{Acknowledgment}

This work is supported in part by the National Natural Science Funds for Young Scholar (no. 51307045), and the authors are indebted to the editor and reviewers for their valuable comments and suggestions.

\section{References}

[1] Q. Zhu, H. Liu, and S. Hu, "Uniformed model of networked control systems with long time delay," Journal of Systems Engineering and Electronics, vol. 19, no. 2, pp. 385-390, 2008.

[2] N. Li and H. Sun, "Passive control of a class of network control systems with long time delay," Journal of Shenyang Jianzhu University, vol. 23, no. 6, pp. 1044-1048, 2007.

[3] W. Xing, J. Wang, and G. Wang, "Stability analysis of networked control system with long-time delay and data packet dropout," 
Journal of Northeastern University, vol. 29, no. 10, pp. 1393-1397, 2008.

[4] Y. Wang and Q. Zhu, " $H_{\infty}$ control of networked control systems with long time delay," Journal of Huazhong University of Science and Technology, vol. 37, no. 9, pp. 60-63, 2009.

[5] Y. Zhang, G. Y. Tang, and N. P. Hu, "Non-fragile control for nonlinear networked control systems with long time-delay," Computers \& Mathematics with Applications, vol. 57, no. 10, pp. 1630-1637, 2009.

[6] Y. Zhang, G. Y. Tang, and Y. Zhao, "Cost-guaranteed $H$-infinity control for a class of nonlinear networked control systems with long time-delay," Control Theory \& Applications, vol. 26, no. 7, pp. 800-804, 2009.

[7] C. Xie and W. Hu, "Analysis and design of a class of networkedcontrol systems with long time-delay and data-packet-dropout," Control Theory \& Applications, vol. 27, no. 9, pp. 1207-1213, 2010.

[8] Q. Wei and W. Wang, "Research on fuzzy self-adaptive PISmith control in long time-delay system," The Journal of China Universities of Posts and Telecommunications, vol. 18, no. 5, pp. 114-117, 2011.

[9] X. Li, X. Wu, W. Hu, and W. Fan, "Fault detection for networked control systems with long time-delay of asynchronous clock," Journal of Nanjing University of Science and Technology, vol. 33, no. 2, pp. 172-182, 2009.

[10] Y. Cai, N. Pan, and X. Xu, " $H_{\infty}$ filtering for networked control systems with long time-delay and data packet dropout," Control and Decision, vol. 25, no. 12, pp. 1826-1836, 2010.

[11] S. G. Wang, "Non-fragile $H_{\infty}$ control with pole constraints for a class of nonlinear sample-data system," in Advanced Electrical and Electronics Engineering, vol. 87 of Lecture Notes in Electrical Engineering, pp. 587-594, Springer, Berlin, Germany, 2011.

[12] S. G. Wang and J. F. Wu, "Observer-based non-fragile $H_{\infty}$ control for a class of uncertain time-delay sampled-data systems," Systems Engineering and Electronics, vol. 33, no. 6, pp. 1352-1357, 2011.

[13] S. G. Wang and J. F. Wu, "Non-fragile $H_{\infty}$ filtering for a class of sampled-data system with long time-delay," ICIC Express Letters B: Applications, vol. 2, no. 6, pp. 1447-1452, 2011.

[14] B. Barmish, "Necessary and sufficient conditions for quadratic stabilizability of an uncertain system," Journal of Optimization Theory and Applications, vol. 46, no. 4, pp. 399-408, 1985.

[15] A. Albert, "Conditions for positive and non-negative definiteness in terms of pseudoinverses," SIAM Journal on Applied Mathematics, vol. 17, no. 2, pp. 434-440, 1969.

[16] X. Tang and J. Yu, "Stability analysis of discrete-time systems with additive time-varying delays," International Journal of Automation and Computing, vol. 7, no. 2, pp. 219-223, 2010. 


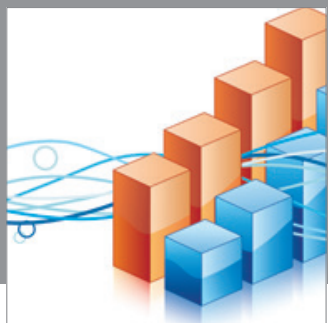

Advances in

Operations Research

mansans

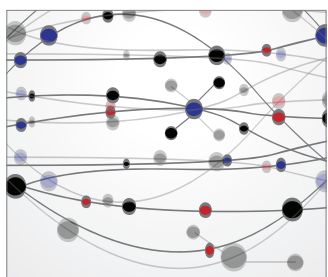

The Scientific World Journal
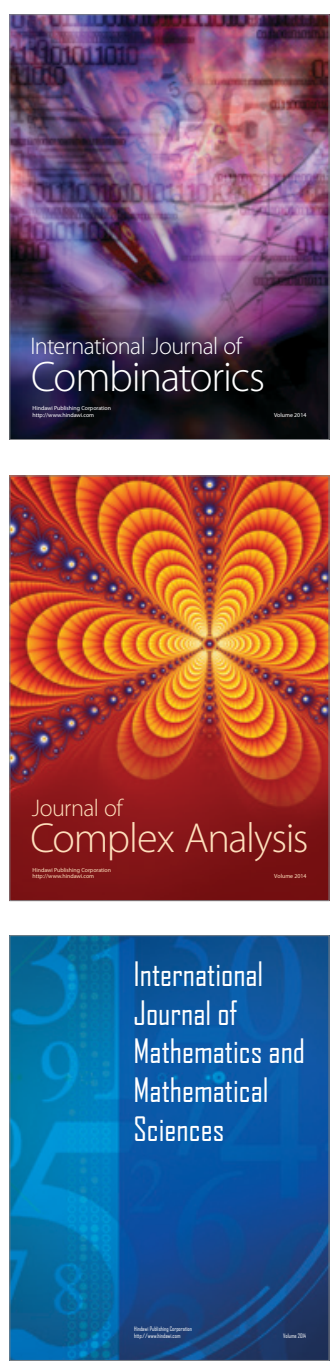
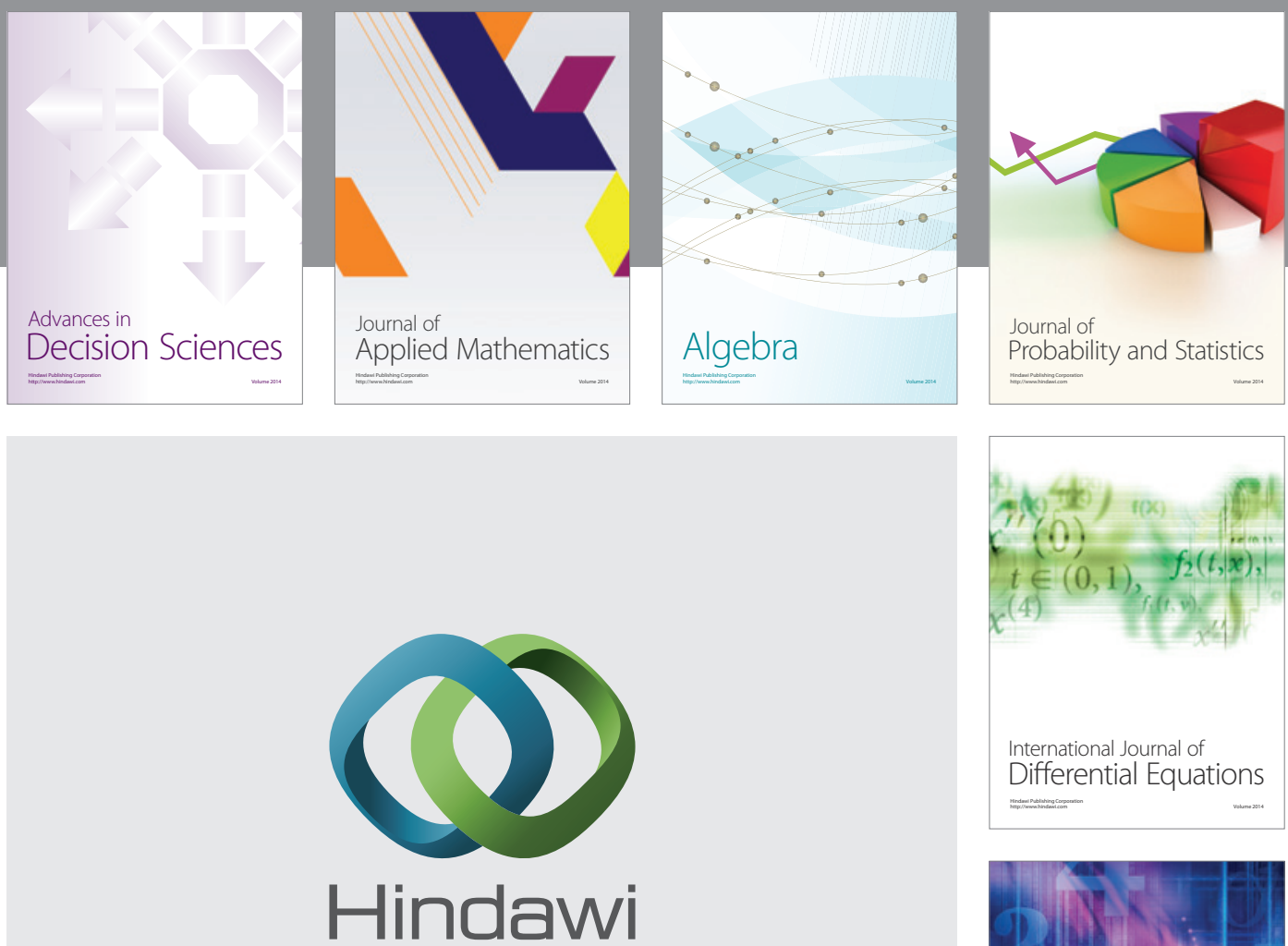

Submit your manuscripts at http://www.hindawi.com
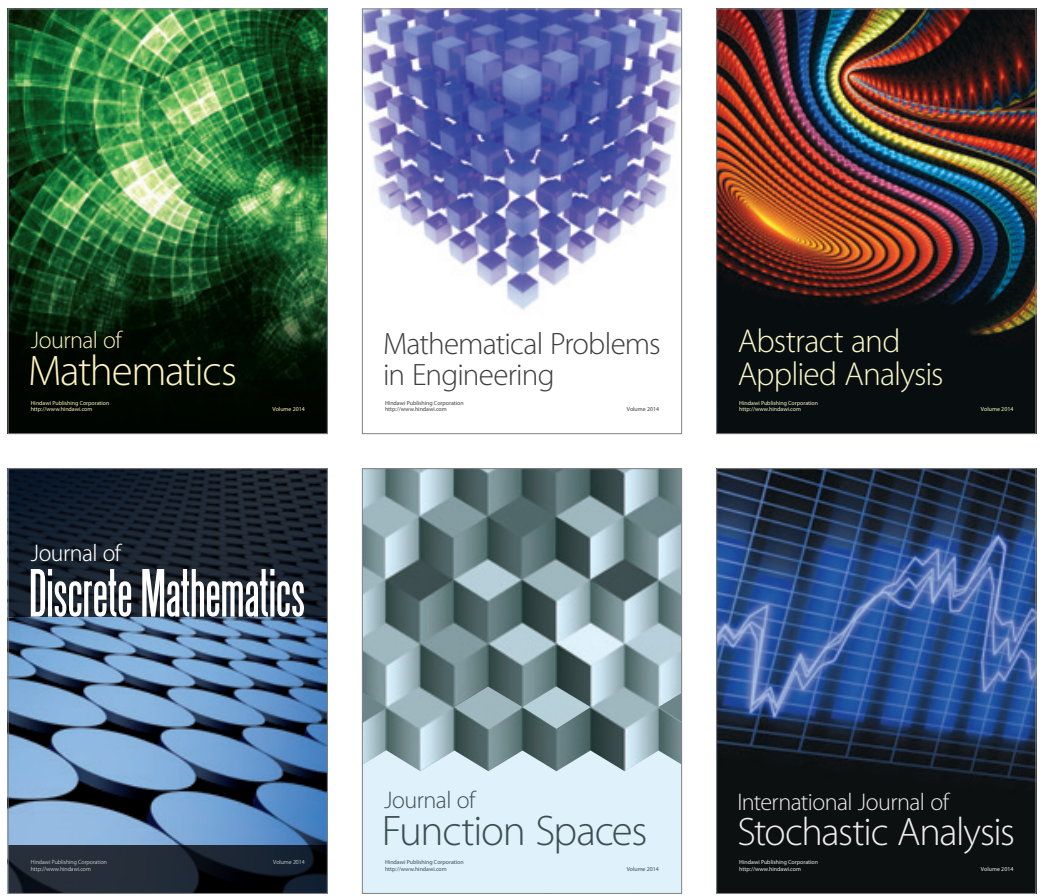

Journal of

Function Spaces

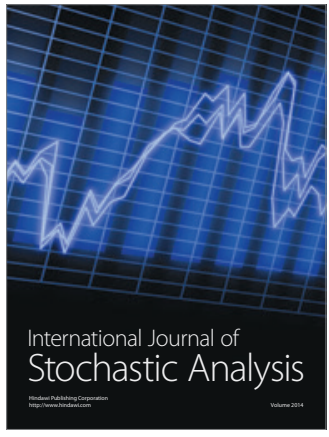

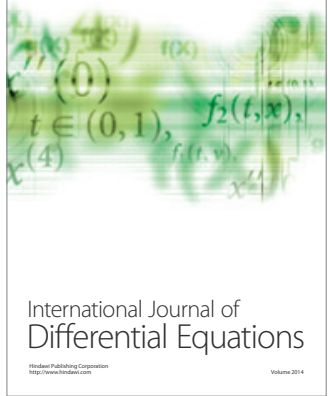
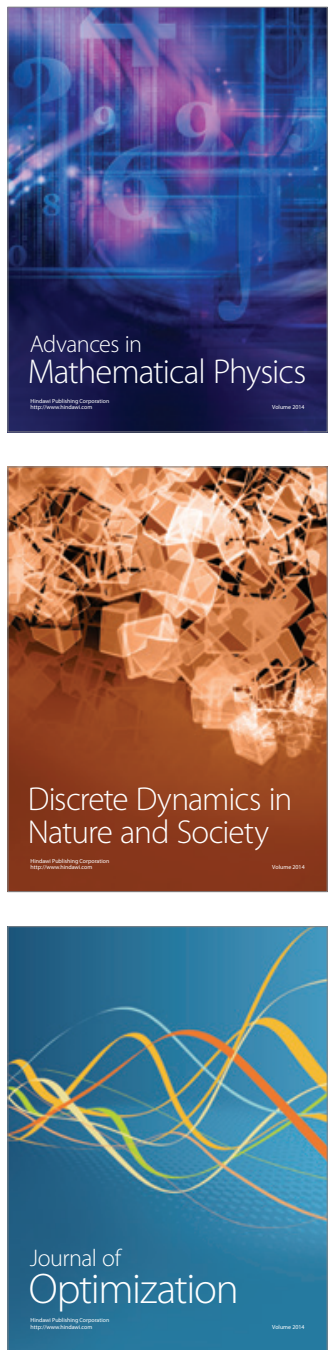